\title{
ON SURFACES IN SPACE OF $r$ DIMENSIONS
}

BY B. C. WONG

Consider a surface $F^{n}$ of order $n$ in $r$-space. Let it be the complete intersection of $q \leqq r-2$ varieties $V_{k_{1}}^{n_{1}}, V_{k_{n}}^{n_{2}}, \cdots, V_{k_{q}}^{n_{q}}$ of orders $n_{1}, n_{2}, \cdots, n_{q}$ and of dimensions $k_{1}, k_{2}, \cdots, k_{q}$, respectively, where

$$
\begin{aligned}
& 3 \leqq k_{1}, k_{2}, \cdots, k_{q} \leqq r-2, \\
& k_{1}+k_{2}+\cdots+k_{q}=r(q-1)+2 .
\end{aligned}
$$

Project $F^{n}$ on an $S_{3}$. The projection $F^{\prime n}$ has a number of characteristics of which we note the following six: $n$, its order; $a$, the order of its tangent cone; $b$, the order of its double curve; $j$, the number of its pinch-points; $t$, the number of its triple points; and $m$, its class. If we project $F^{n}$ on an $S_{4}$, the projection has a finite number, $d$, of improper double points. We shall call these seven characteristics, of which $n, a, t, m$ are often regarded as essential, the characteristics of $F^{n}$, and they are known to satisfy the following relations:*

$$
\begin{aligned}
a+2 b & =n(n-1), \quad j+2 d=n(n-1)-a, \\
j & =\frac{1}{4}[a(3 n-4)-n(n-1)(n-2)+6 t-2 m], \\
d & =\frac{1}{8}[n(n-1)(n+2)-3 a n-6 t+2 m] .
\end{aligned}
$$

For $r=5, q=3, k_{1}=k_{2}=k_{3}=4, F^{n}$ is the intersection of three hypersurfaces in $S_{5}$. Formulas for its characteristics are known $\dagger$ and they are symmetric functions of the orders of the hypersurfaces. In this note we present analogous formulas for the same characteristics of $F^{n}$ for $r$ general and for $q \leqq r-2$. As the method of obtaining these formulas is familiar and has been applied by the writer time and again to similar enumerative problems, $\ddagger$ we shall here omit all demonstration.

* Severi, Intorno ai punti doppi impropri di una superficie generale dello spazio a quattro dimensioni, e a'suoi punti tripli apparenti, Rendiconti di Palermo, vol. 15 (1901), pp. 33-51.

$\dagger$ B. C. Wong, On surfaces in spaces of four and five dimensions, this Bulletin, vol. 36 (1930), pp. 681-686. Opportunity is here taken to correct an error in the formula for $T^{\prime \prime}$ on page 685 of this paper. The formula should read

$$
T^{\prime \prime}=\frac{1}{2} \lambda \mu \nu(\lambda-1)(\mu-1)(\nu-1)(\mu \nu+\nu \lambda+\lambda \mu-2 \lambda-2 \mu-2 \nu) \text {. }
$$

$\ddagger$ B. C. Wong, loc. cit., and also the paper On the number of apparent double points of $r$-space curves, this Bulletin, vol. 37 (1931), pp. 421-423. 
If $q=r-2$, and, from (A), $k_{1}=k_{2}=\cdots=k_{r-2}=r-1, F^{n}$ is the complete intersection of $r-2$ hypersurfaces in $S_{r}$. The formulas for its characteristics are

$$
\begin{aligned}
n= & n_{1} n_{2} \cdots n_{r-2}, \\
a= & n\left(\sum n_{i}-r+2\right), \\
b= & \frac{1}{2} n\left(n-\sum n_{i}+r-3\right), \\
d= & \frac{1}{2} n\left[n-\sum n_{i} n_{j}+(r-4) \sum n_{i}-\frac{1}{2}(r-3)(r-4)\right], \\
j= & n\left[\sum n_{i} n_{j}-(r-3) \sum n_{i}+\frac{1}{2}(r-2)(r-3)\right], \\
t= & \frac{1}{6} n\left[n\left(n-3 \sum n_{i}\right)+3(r-3)\left(n-2 \sum n_{i}\right)\right. \\
& \left.+2\left(\sum n_{i}{ }^{2}+3 \sum n_{i} n_{j}\right)+(r-3)(3 r-8)\right], \\
m= & n\left[\sum\left(n_{i}-1\right)^{2}+\sum\left(n_{i}-1\right)\left(n_{j}-1\right)\right], \quad(i \neq j) .
\end{aligned}
$$

Now if $q \leqq r-2$, one or more of the $k$ 's will be less than $r-1$. Let the $i$ th variety $V_{k_{i}}^{n_{i}}$ be intersected by a general $S_{r+2-k_{i}}$ in a surface $F^{n_{i}}$. We assume known the characteristics $a_{i}, b_{i}, t_{i}$ of $F^{n^{i}}$ besides $n_{i}$. The characteristics of $F^{n}$ are given by the following formulas which are functions of $n_{i}$ and $q$, and also of $a_{i}, b_{i}$ and $t_{i}$ :

$$
\begin{aligned}
n= & n_{1} n_{2} \cdots n_{q}, \\
a= & n\left(\sum n_{i}-q\right)-2 n \sum b_{i} / n_{i}=n \sum a_{i} / n_{i}, \\
b= & \frac{1}{2} n\left(n-\sum n_{i}+q-1\right)+\sum b_{i} / n_{i}=\frac{1}{2} n(n-1)-\frac{1}{2} n \sum a_{i} / n_{i}, \\
d= & \frac{1}{2} n\left[n-\sum n_{i} n_{j}+(q-2) \sum n_{i}-\frac{1}{2}(q-1)(q-2)\right] \\
& +n \sum_{i}\left(\sum n_{j}-q+1\right) b_{i} / n_{i}-2 n \sum b_{i} b_{j} / n_{i} n_{j}, \\
j=n & {\left[\sum n_{i} n_{j}-(q-1) \sum n_{i}+\frac{1}{2} q(q-1)\right] } \\
& -2 n \sum_{i}\left(\sum n_{j}-q\right) b_{i} / n_{i}+4 n \sum b_{i} b_{j} / n_{i} n_{j}, \\
t= & \frac{1}{6} n\left[n\left(n-3 \sum n_{i}\right)+3(q-1)\left(n-2 \sum n_{i}\right)\right. \\
& \left.+2\left(\sum n_{i}{ }^{2}+3 \sum n_{i} n_{j}\right)+(q-1)(3 q-2)\right]+n \sum t_{i} / n_{i} \\
& +n \sum_{i}\left[n-2 \sum n_{j}-n_{i}+2(q-1)\right] b_{i} / n_{i}+4 n \sum b_{i} b_{j} / n_{i} n_{j}, \\
m= & n\left[\sum\left(n_{i}-1\right)^{2}+\sum\left(n_{i}-1\right)\left(n_{j}-1\right)\right]+3 n \sum t_{i} / n_{i} \\
& -n \sum_{i}\left(2 \sum n_{j}+3 n_{i}-2 q+2\right) b_{i} / n_{i}+4 \sum b_{i} b_{j} / n_{i} n_{j}, \\
& \quad(i \neq j) .
\end{aligned}
$$

All the formulas of each of these two sets satisfy relations (B).

University of CaLIFornia 